\title{
The Effect of Plantar Flexor Fatigue and Cognitive Recall Task on Standing Balance
}

\author{
William J. Smith ${ }^{1}$, Dillon J. Richards ${ }^{1}$, Shibo H. Zhou ${ }^{1}$, Tanner Kennedy ${ }^{1}$ \\ University of Western Ontario
}

Introduction: Previous studies have shown that fatigue of the plantar flexor muscles and mental tasks each can lead to impaired balance. The purpose of this study is to analyze the effects of a combined increase in cognitive load and plantar flexor fatigue can have on standing balance. Methods: Fifteen (7 males, 8 females) healthy individuals participated in one testing session. Prior to testing, participants were screened for existing balance and plantar flexor health issues via a medical history questionnaire. Participants performed a total of twelve standing balance trials, each 30 seconds in duration. These trials consisted of three control, three recall, three fatigue, and three fatigue recall trials. For each trial, participants closed their eyes and stood with their feet together on a force plate. Recall ability was evaluated based on the participant's ability to accurately complete the reverse digit recall section of the SCAT-3. Plantar flexor fatigue was achieved by performing five repetitions of standing calf raises. Each repetition consisted of a five second eccentric contraction, a five second concentric contraction, and a five second isometric contraction. The means of the $90 \%$ radius of the center of pressure was compared to the standing baseline across all conditions.

Results: Similar significant differences were found across $90 \%$ radius and center of pressure in both medial-lateral and anterior-posterior directions. Across all three variables, the recall condition was not significantly different $(p>0.05)$ than control standing. Both fatigue and fatigue recall conditions were significantly different $(p<0.05)$.

Conclusion: Collectively, the data indicates that fatigue, rather than recall, had a greater effect on all measured variables of balance. This suggests that balance is a primarily muscle driven task, and not a mental task.

\section{Introduction}

Balance is defined as the dynamic movement of body posture to prevent falling (1). This posture requires an object's center of mass (CoM) to remain within its base of support (BoS) (2). If an inanimate objects CoM falls outside of its BoS, it is considered unstable and will fall over (2). Fortunately, humans have the ability to produce muscle forces to counteract the force of gravity and prevent them from becoming unstable and falling (2). Interestingly, it has been determined that standing balance is achieved about the ankle joint mainly through contraction of the plantar flexors (3). Minimal contraction of the dorsi flexors are necessary (3).

Despite balance regulation being considered an automatic task, it has been shown to require attentional resources (4). The cognitive demand required for balancing increases as the amount of sensory information regarding the bodies location in space decreases (5). In situations where multitasking is required, individuals may have difficulty allocating the necessary cognitive resources to maintain standing balance (5). For example, the addition of both an auditory and visuospatial tasks to quiet standing increased postural sway in healthy adults (5).

Several studies have demonstrated the negative effects that plantar flexor fatigue has on the ability to maintain standing balance (6-8). Plantar flexor fatigue was shown to create both short term and long term postural instability in the AnteriorPosterior (AP) direction in healthy adults (6), as well as university age individuals (7). Fatigue must be localized to muscles involved in postural support, for example plantar flexors, rather than a general state of fatigue (8). This reduction in postural control ability could cause a decrease in performance and place individuals at an increased risk of injury. However, few studies have examined the effects that an increase in cognitive load may have on postural control while the plantar flexors are in a fatigued state. Understanding this relationship in a healthy population may be helpful in creating a standard for 
studies examining a population with specific neurological or physical deficits to refer to. This study will also provide significant explanation of possible balance deficiencies in activities that are both physical and cognitive in nature.

The purpose of this study was to analyze the effects that increasing cognitive load and plantar flexor fatigue have on postural sway. Force plates were used to measure the degree of movement of the participants' center of mass. A standardized cognitive recall task was used to increase cognitive load, and a calf raise protocol was used to fatigue the planter flexors. It was hypothesized that increasing both cognitive load and fatigue levels would show a greater change in postural sway in the AP direction than in the Medial-Lateral (ML) direction. An increase in both recall and fatigue conditions simultaneously would result in the greatest amount of postural sway.

\section{Methods}

Participants: Fifteen healthy individuals (7 males, 8 females) between the ages of 18-53 years participated in this study. Participants volunteered after seeing recruitment posters that were administered on campus at Western University. Prior to completing the testing protocol, all participants filled out a questionnaire that would identify any plantar flexor or balance issues that could preclude them from participating. No participants were excluded. Every participant provided informed consent before taking part in the testing protocol.

Measurements: Postural sway was measured by examining participant's center of pressure (CoP) while standing on a Kistler force plate. The force plate measured each participant's $\mathrm{CoP}$ in both the AP direction and $\mathrm{ML}$ direction. Signals from the force plate were amplified (AMP 9865C, Kistler Holding AG, Winterthur, Switzerland) and sampled at $100 \mathrm{~Hz}$ using a 16-bit analogue-todigital conversion board (779407-01, National Instruments, Austin, TX, USA). Data was recorded using a custom LabVIEW program (Version 10.0, National Instruments, Austin, TX, USA). Cognitive demands were measured by assessing the participant's ability to successfully perform a reverse digit recall task using the Digit Backward portion of the Sport Concussion Assessment Tool- $3^{\text {rd }}$ Edition (SCAT-3) (Figure 1).

Protocol: For all trials, participants remained as still as possible with their feet together, their arms at their side, and their eyes closed. Participants underwent four conditions: control (S), recall $(R)$, fatigue $(F)$, and fatigue recall $(F R)$.

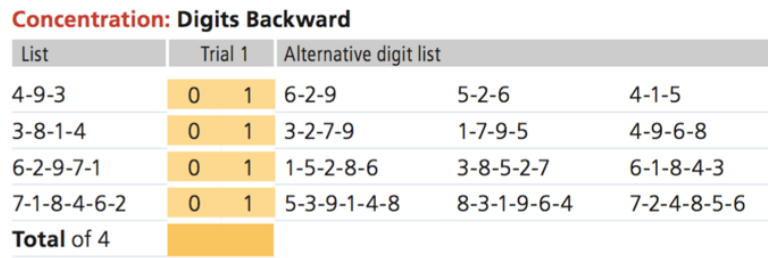

Figure 1. Concentration: Digits Backwards. Adapted from British Journal of Sports Medicine. 2017, Retrieved from http://bjsm.bmj.com/content/ bjsports/47/5/259.full.pdf

Each condition was performed three times for a total of twelve trials. Each trial was thirty seconds in length with an allotted ten second break between each. The control condition determined each participant's natural level of postural sway. In the recall condition, participants performed the reverse digit recall task outlined in the SCAT-3 while trying to maintain balance. This task was selected as there is no known practice effect (19). For each trial, participants began by repeating a three-digit span with an extra digit being added after each successful recall. If participants progressed to the six-digit span they were asked to continue to repeat a different six-digit sequence until the time was up. In the fatigue condition, participants achieved plantar flexor fatigue by performing five sets of five reps of calf raises. Each rep was comprised of a five second concentric, isometric, and eccentric contraction. Balance was measured immediately after completing the exercise to maximize the effect of the plantar flexor fatigue. Participant's repeated the fatigue task before beginning each subsequent trial. The fatigue recall condition consisted of both the fatigue task and the recall task. Participants performed the same fatigue protocol prior to each trial. During the trial, participants were assessed on their ability to perform a reverse digit recall task. Scores were determined using the number of correct answers/total responses.

Calculations: Using ground reaction force (GRF) and moment (M) data collected from the force plate, CoP was calculated in both the ML and AP directions using the following formulas:

$$
\begin{aligned}
& \text { absolute } \mathrm{CoP}_{\mathrm{ML}}=\frac{M_{y}+c * F_{x}}{F_{z}} \\
& \text { absolute } \mathrm{CoP}_{\mathrm{AP}}=\frac{M_{x}+c * F_{y}}{F_{z}}
\end{aligned}
$$

where $c$ is a constant that represents the location of the origin of the force plate $(0.0388 \mathrm{~m})$. 
In order to normalize the data to an origin of $(0,0)$, the following equation was used:

normalized CoP $\mathrm{PL}_{\mathrm{ML}}=$ absolute $\mathrm{CoP}_{\mathrm{ML}}-$ mean $\mathrm{CoP}_{\mathrm{ML}}$

The same equation was used to calculate the normalized $\mathrm{CoP}_{\mathrm{AP}}$. The normalized data was used for the rest of the calculations.

The following equation was used to calculate range in both $\mathrm{AP}$ and $\mathrm{ML}$ directions:

$$
\text { range } \mathrm{CoP}=\mathrm{CoP}_{\max }-\mathrm{CoP}_{\min }
$$

In order to calculate a circle with a radius that encompasses $90 \%$ of the data, a distance from the origin $(0,0)$ was calculated using the following equation for each data point:

$$
\text { distance from centre }=\sqrt{\mathrm{CoP}_{\mathrm{ML}}{ }^{2}+\mathrm{CoP}_{\mathrm{AP}}{ }^{2}}
$$

Finally, the Microsoft Excel function "PERCENTILE.EXC" was used to calculate the $90^{\text {th }}$ percentile value within the array of distance from centre data points from 0 to 30 seconds. The calculated value corresponds to the radius of a circle that encompasses $90 \%$ of the values.

Data Analysis: Within each experimental condition, the individual's three trials were averaged to produce a mean value for range of $\mathrm{CoP}_{\mathrm{ML}}$, $\mathrm{COP}_{\mathrm{AP}}$, and the radius of a circle that encompasses $90 \%$ of the CoP data points. Further quantitative analysis was done to produce a mean and standard deviation between all subjects for each of the four conditions for all variables measured. A similar method was done on the success rates of the reverse digit recall task in order to produce a mean and standard deviation for the recall and fatigue recall conditions.

T-tests were performed using the paired 2tailed method. An alpha value of 0.05 was assigned as a determinant of significance for all analysis. Ttests were done between each of the four experimental conditions to determine any significant differences. A total of six t-tests ( $S$ vs. R, $S$ vs. F, S vs. $F R, R$ vs. $F, R$ vs. $F R$ and $F$ vs. FR) were done per variable. A similar t-test was done to compare the success rate of the reverse digit recall task for the $R$ vs. FR conditions.

\section{Results}

The overall results are summarized within

\begin{tabular}{|c|c|c|c|c|}
\hline & Standing & Recall & Fatigue & $\begin{array}{c}\text { Fatigue } \\
\text { Recall }\end{array}$ \\
\hline $\begin{array}{l}\mathrm{CoP}_{\mathrm{ML}} \\
\text { Range (m) }\end{array}$ & $\begin{array}{l}0.0245 \pm \\
0.0127 \\
\end{array}$ & $\begin{array}{l}0.0247 \pm \\
0.0105\end{array}$ & $\begin{array}{l}0.0385 \pm \\
0.0172 \\
\end{array}$ & $\begin{array}{l}0.0369 \pm \\
0.0213\end{array}$ \\
\hline $\begin{array}{l}\mathrm{CoP}_{\mathrm{AP}} \\
\text { Range (m) }\end{array}$ & $\begin{array}{l}0.0330 \pm \\
0.00849\end{array}$ & $\begin{array}{l}0.0399 \pm \\
0.0261\end{array}$ & $\begin{array}{l}0.0512 \pm \\
0.0214\end{array}$ & $\begin{array}{l}0.0550 \pm \\
0.0412\end{array}$ \\
\hline $\begin{array}{l}90 \% \\
\text { Radius } \\
\text { (m) }\end{array}$ & $\begin{array}{l}0.0112 \pm \\
0.00436\end{array}$ & $\begin{array}{l}0.0118 \pm \\
0.00517\end{array}$ & $\begin{array}{l}0.0167 \pm \\
0.00614\end{array}$ & $\begin{array}{l}0.0170 \pm \\
0.0109\end{array}$ \\
\hline
\end{tabular}
Table 1. The recall protocol did not produce any
Table 1. Subject's $(n=15)$ mean \pm standard deviation values for variables measured in all experimental conditions.

statistically significant differences in $90 \%$ radius $(p=0.62), M L$ range $(p=0.90)$ and AP range $(p=0.28)$ compared to baseline. When compared to standing, the fatigue condition produced a significantly different $90 \%$ radius $(p=0.003), M L$ range $(p=0.0083)$ and AP range $(p=0.0045)$. Fatigue was also significantly different than the recall condition in $90 \%$ radius $(p=0.00075)$, ML range $(0.0034)$ but was not significantly different in AP range $(p=0.09)$. Similar results were found in the fatigue recall condition which was significantly different than standing in $90 \%$ radius $(p=0.04), M L$ range $(p=0.022)$ and $A P$ range $(p=0.044)$. In addition, fatigue recall was significantly different than the recall condition in $90 \%$ radius $(p=0.006), M L$ range $(p=0.021)$ and $A P$ range $(p=0.0096)$. Finally, there were no significant differences between the fatigue and fatigue recall conditions in $90 \%$ radius $(p=0.87), M L$ range $(p=0.74)$ and AP range $(p=0.67)$. A summary of the data can be found in Figures 2-4.

In addition, the success rate of the reverse digit recall task was $61 \% \pm 13.6 \%$ for the recall alone condition and $65 \% \pm 17.2 \%$ for the fatigue recall condition. There was not a significant difference in the success rate between recall and fatigue recall conditions $(p=0.24)$.

\section{Discussion}

This study analyzed the effects that cognitive recall tasks and plantar flexor fatigue have on standing balance. It was hypothesized that an increase in postural sway in the AP direction would be found in both the fatigue and recall conditions, with the largest increase occurring in the fatigue recall condition. Our results showed that the fatigue condition had a significant increase in postural sway, whereas the recall condition did not. The fatigue recall condition had significantly more sway than standing, but not more sway than the fatigue 


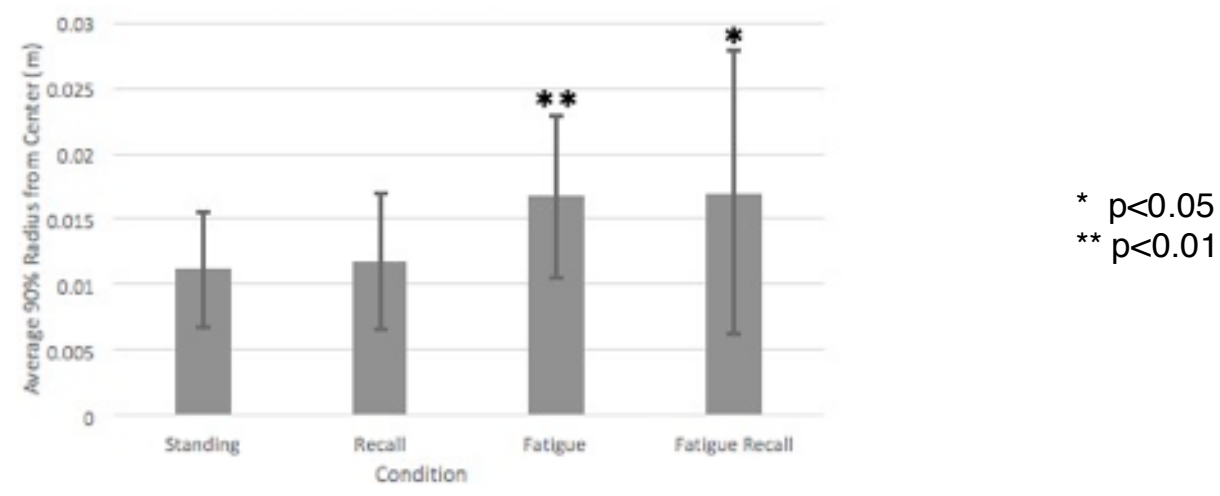

Figure 2. Mean radius (SD) in meters that encompasses $90 \%$ of CoP (90\% radius) data points for each of the 4 experimental conditions. Significance is compared to standing balance

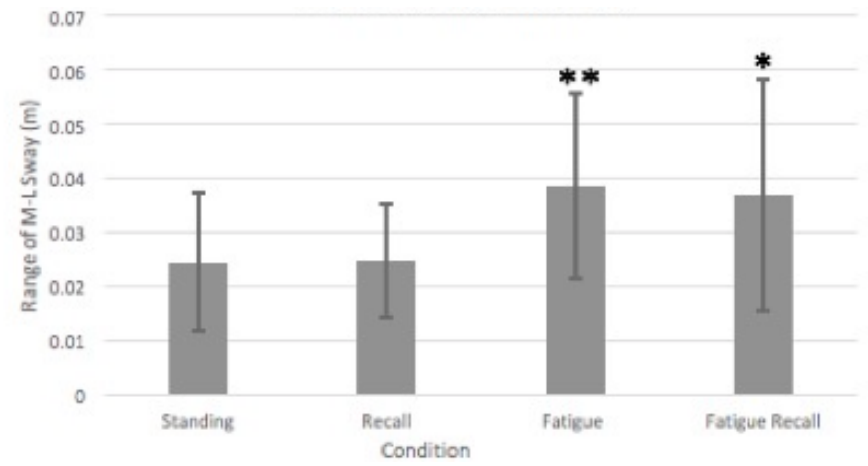


condition. The results do not support the original hypothesis.

The lack of a statistically significant difference between the fatigue and recall conditions, and the fatigue and fatigue recall conditions, indicate that participants sway was a direct result of plantar flexor fatigue. All balance trials that followed the fatigue protocol had no statistically significant difference from each other, regardless if there was a digit recall component. This shows that all of the balance detriments were directly resulting from fatigue protocol.

The current study reported that postural sway was significantly increased in the AP and the $M L$ directions, which also resulted in a significantly increased $90 \%$ radius in fatigued participants. Previous authors have reported an increase in postural sway when the plantar flexor muscles were fatigued $(4,6-8)$. Plantar flexor fatigue has been shown to result in an increase in postural sway only in the AP direction $(7,9)$. Postural control in the AP direction is dominated by the plantar and dorsi flexors, whereas control in the ML direction is dominated by muscles about the hip (9). It is possible that the fatigue protocol is responsible for the increase in ML sway as the protocol did not isolate the fatigue to muscles that solely act as plantar flexors like the gastrocnemius and soleus. Muscles such as the peroneus longus (primarily an evertor) and the tibialis posterior (primarily an invertor) have been shown to have a secondary plantar flexor function during a calf raise task (10). Although these muscles are not primarily used to stabilize the CoP in the ML direction, it is possible that their fatigue resulted in enough instability to cause the reported sway. Previous research has determined that fatiguing muscles that are not involved in quiet stance has no effect on postural sway $(8,11)$. This suggests that the differences found in postural sway are directly caused by the localized fatigue of the plantar flexors and not by being in a general state of fatigue. This may occur due to the fatigued muscles inability to generate the necessary compensatory contractions normally seen in the ankle strategy (3).

The introduction of a recall task did not have a significant effect on postural sway in this study. As such, we determined that the attentional resources dedicated to balancing were not interrupted by the addition of the cognitive tasks. This result has not been universally accepted. Vuillerme et al. examined the effects of a reaction time task on standing balance and found a significant increase in attentional demands during quiet standing. Their study resulted in no change of sway, but an increase in the time that it took to perform the reaction time task. This result led them to conclude that when fatigued, there must be some conscious attentional demands necessary to maintain balance (4). The differences in results may be due to the nature of the task. The digit backwards recall task analyzed in the present study may not have been difficult enough draw resources from cognitive performance.

The dual task protocol was employed to increase the neural demands the participants were under during standing balance. Ouchi et al. found that standing balance was regulated by activation of the cerebellar vermis as well as the right occipital lobe, primarily the visual cortex (12). The vermis and surrounding area activate the fastigial nuclei, the pathway for activation of extensor muscles, the primary group involved in standing (12). However, recent studies have found that higher brain areas of the cortex are also activated during standing balance which include the dorsolateral prefrontal cortex (DLPFC) (13). Reverse Digit Recall tasks have been seen to activate these same areas of the brain, primarily the DLPFC $(14,15)$. Therefore, both tasks require input from similar brain areas and should create exceeding demand. However, the lack of balance deficits seen in this study may be a function of the simplicity of the recall task. Working memory has been defined as having a limit of $7 \pm 2$ units of information (16). The reverse digit span has a maximum of 6 units and may not have taxed the prefrontal cortex enough. Balance requirements may have been shifted partially from the DLPFC to more primitive structures such as the cerebellum.

Limitations: The difficulty of the recall task that was chosen may have limited the results of the study. Some participants were unable to complete the task due to the time limit of 30 seconds per trial. Thus, it can be assumed that the task was not difficult enough to place a significant cognitive load on the participants. It is encouraged that further research be done using a more cognitively challenging task. Additionally, the fatigue protocol didn't include a way of measuring the participants level of fatigue. This could mean that certain participants experienced a greater level of fatigue that others.

The population of this study consisted of healthy young adults with no history of balance related or cognitive issues. Knowing that the ability to maintain balance diminishes with age may limit the validity of this study when applied to older adults (17). While the Reverse Digit Recall did not appear to place a large enough cognitive load on the subjects tested, the same task may have been challenging to an elderly subject with cognitive impairments to the working memory system. Within the elderly population, age-related memory loss is a symptom of 
a preclinical Alzheimer's disease pathological state. Neuron damage within the hippocampus results in a decreased capacity for working memory (18). The recall task used would likely have been more taxing to the DLPFC and may lead to different results than the study presented. The results of this study should be used with caution when applied to individuals with certain physical or neurocognitive deficits (17).

Future implications: Future research could utilize multiple cognitive tasks of varying difficulty allowing analysis of the direct effect cognitive tasks have on standing balance. Although taxing specifically plantar flexors may allow for analysis of specific effects, it lacks real world implications.

\section{Conclusion}

In summary, our results show that muscular fatigue has a larger effect on standing balance than simple mental tasks. AP sway, ML sway, and $90 \%$ radius were all found to increase as a result of fatigue. However, no statistically significant differences were found on performance of the recall task or on balance when recall was employed. Future studies should focus on pathological populations, which may be more sensitive to the measures used.

\section{References}

1. Winter, DA. Human balance and posture control during standing and walking. Gait Posture. 1995; 3(4):193-214. DOI: 10.1016/0966-6362(96)82849-9

2. Pollock, AS, Durward, BR, Rowe, PJ, Paul, JP. What is balance? Clin Rehabil. 2000; 14(4):402-406. DOI: 10.1191/0269215500cr342oa

3. Gatev, P, Thomas, S, Kepple, T, Hallett, M. Feedforward ankle strategy of balance during quiet stance in adults. J Physiol, 1999; 514(3):915-928. DOI: 10.1111/j.14697793.1999.915ad.x

4. Vuillerme, N, Forestier, N, Nougier, V. Attentional demands and postural sway: the effect of the calf muscles fatigue. Med Sci Sports Exerc. 2002; 34(12):1907-1912. DOI: 10.1249/01.MSS.0000039062.36895.AB

5. Shumway-Cook, A, Woollacott, M. Attentional demands and postural control: the effect of sensory context. J Gerontol A Biol Sci Med Sci. 2000; 55(1):M10-M16.

6. Gimmon, Y, Riemer, R, Oddsson, L, Melzer, I. The effect of plantar flexor muscle fatigue on postural control. J Electromyogr Kinesiol. 2011; 21(6):922-928. DOI: 10.1016/j.jelekin.2011.08.005

7. Grey, T, Redguard, D, Wengle, R, Wegscheider, P. Effect of plantar flexor muscle fatigue on postural control. WURJ: Health and Natural Sciences. 2013; 4(1):Article 1. DOI: http://dx.doi.org/10.5206/wurjhns.2013-14.1

8. Hlavackova, P, Pradon, D, Vuillerme N. Control of bipedal posture following localised muscle fatigue of the plantarflexors and finger-flexors. Eur J Appl Physiol. 2012; 112(2): 789-793. doi:10.1007/s00421-011-2009-x

9. Winter, DA, Prince, F, Frank, JS, Powell, C, Zabjek, KF. Unified theory regarding $A / P$ and $M / L$ balance in quiet stance. J Neurophysiol. 1996; 75(6):2334-2343.
10. Segal, RL, Song, AW. Nonuniform activity of human calf muscles during an exercise task. Arch Phys Med Rehabil. 2005; 86(10):2013-2017. DOI: 10.1016/j.apmr.2005.04.012

11. Cogswell, FD, Huang, F. Dietze, B. The Effects of Upper-Body and Lower-Body Fatigue on Standing Balance. WURJ: Health and Natural Sciences. 2017; 7(1):Article 12. DOI: http://dx.doi.org/10.5206/wurjhns.2016-17.11

12. Ouchi, Y, Okada, H, Yoshikawa, E, Nobezawa, S, Futatsubashi, M. Brain activation during maintenance of standing postures in humans. Brain. 1999; 122(2): 329-338.

13. Mihara, M, Miyai, I, Hatakenaka, M, Kubota, K, Sakoda, S. Role of the prefrontal cortex in human balance control. Neuroimage. 2008; 43(2):329-336. DOI: 10.1016/j.neuroimage.2008.07.029

14. Hoshi, Y, Oda, I, Wada, Y, Ito, Y, Yamashita, Y, Oda, M, Ohta, $\mathrm{K}$, Yamada, $\mathrm{Y}$, Tamura, M. Visuospatial imagery is a fruitful strategy for the digit span backward task: a study with nearinfrared optical tomography. Brain Res Cogn Brain Res. 2000; 9(3):339-342.

15. Sun, $X$, Zhang, $X$, Chen, $X$, Zhang, $P$, Bao, M, Zhang, D, Chen, J, He, S, Hu, X. Age- dependent brain activation during forward and backward digit recall revealed by fMRI. Neuroimage. 2005; 26(1):36-47. DOI: 10.1016/j.neuroimage.2005.01.022

16. Miller GA. The magical number seven plus or minus two: some limits on our capacity for processing information. 1956. Psychol Rev. 1994; 101(2):343-352. DOI:10.1037/0033295X.101.2.343

17. Horak, FB, Shupert, CL, Mirka, A. Components of postural dyscontrol in the elderly: a review. Neurobiol Aging. 1989; 10(6);727-738. DOI: 10.1016/0197-4580(89)90010-9

18. Chandler, MJ, Lacritz, LH, Cicerello, AR, Chapman, SB, Honig LS, Weiner, MF, Cullum, CM. Three-word recall in normal aging. J Clin Exp Neuropsychol. 2004; 26(8):1109-1120. DOI: $10.1080 / 13803390490515540$

19. Valovich TC, Perrin DH, Gansneder BM. Repeat administration elicits a practice effect with the Balance Error Scoring System but not with the Standardized Assessment of Concussion in high school athletes. Journal of athletic training. 2003 Jan;38(1):51. 